\title{
Conforto térmico em espaços públicos de passagem: estudos em ruas de pedestres no estado de São Paulo
}

Thermal comfort in public open spaces: studies in pedestrian streets in Sao Paulo state, Brazil

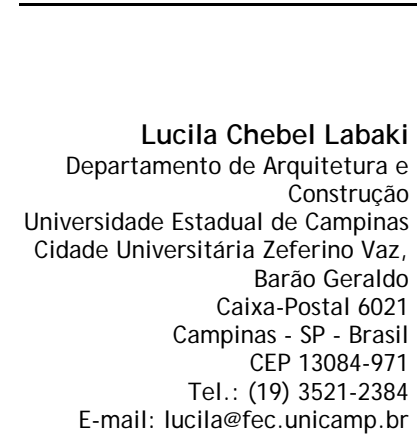

Maria Solange Gurgel de Castro

Fontes

Departamento de Arquitetura Urbanismo e Paisagismo, Faculdade de Arquitetura Artes e Comunicação Universidade Estadual Paulista J úlio de Mesquita Filho AV. Engenheiro Luiz Edmundo Carrijo

Coube, s/ n, Vargem Limpa Caixa-Postal 473 Bauru - SP - Brasil CEP 17033360

Tel.: (14) 221-6059

E-mail: sgfontes@faac.unesp.br

Carolina Lotufo Bueno-

Bartholome

Departamento de Planejamento, Urbanismo e Ambiente, Faculdade de Ciências e Tecnologia

Universidade Estadual Paulista

Campus Presidente Prudente Rua Roberto Simonsen, 305, Vila Santa Helena

Presidente Prudente - SP - Brasi CEP 19060-900

Tel.: (18) 3229-5689

E-mail: carolinalotufo@fct.unesp.br

Cristiane Dacanal Instituto Federal de Educação, Ciência e Tecnologia do Sertão Pernambucano

Estrada do Tamboril, $\mathrm{s} / \mathrm{n}$

Ouricuri - PE - Brasi CEP 56200-000

Tel.: (87) 9930-6637 E-mail: cristiane.dacanal@ifsertaope.edu.br

Recebido em 11/10/11

Aceito em 12/12/11

\section{Lucila Chebel Labaki \\ Maria Solange Gurgel de Castro Fontes \\ Carolina Lotufo Bueno-Bartholomei \\ Cristiane Dacanal}

\section{Resumo}

$\mathbf{E}$

ste artigo apresenta resultados de um estudo sobre conforto térmico em espaços públicos de passagem, em ruas de pedestres nas cidades de Campinas, Bauru e Presidente Prudente, no Estado de São Paulo. O estudo foi desenvolvido dentro de uma pesquisa mais ampla sobre $o$ conforto térmico em diferentes tipos de espaços urbanos abertos, nas mesmas cidades. A metodologia empregada envolveu o monitoramento microclimático (temperatura, temperatura de globo, umidade relativa do ar, velocidade do ar e radiação solar global), em diferentes condições de tempo, e entrevistas estruturadas, para identificar a sensação térmica e as variáveis pessoais dos usuários. A análise dos resultados permitiu identificar diferenças entre a sensação térmica real (ASV) e o conforto calculado pela temperatura fisiológica equivalente (PET). Os limites de conforto térmico variaram entre as cidades: $20-29^{\circ} \mathrm{C}$ para Campinas, $21-30{ }^{\circ} \mathrm{C}$ para Bauru, e $14-24^{\circ} \mathrm{C}$ para Presidente Prudente. Entretanto, a sensação de neutralidade térmica para 59,5\% do total da amostra (308 de 519 indivíduos) foi de 18 a $26^{\circ} \mathrm{C}$. Esses resultados são compatíveis com os limites propostos por Monteiro e Alucci (2007) para a cidade de São Paulo e podem contribuir como parâmetro de avaliação da qualidade térmica de outros espaços públicos de passagem nas mesmas cidades.

Palavras-chave: Espaços públicos urbanos. Conforto térmico. Espaços de passagem.

\section{Abstract}

This paper presents the results of a study on the thermal comfort in open urban spaces, undertaken in pedestrian streets located in the three towns, Campinas, Baurú, and Presidente Prudente, in the state of Sao Paulo. The study was developed as part of a more extensive project on thermal comfort in different kinds of open public spaces in Brazil. The methodology involved monitoring the microclimatic variables (air and globe temperature, humidity, air velocity and global solar radiation), and structured interviews, in order to assess the actual thermal comfort through the Actual Sensation Vote (ASV) and the personal users' variables. The Physiological Equivalent Temperature (PET) was also calculated. The results show different limits for neutral temperature in each city: $20-29^{\circ} \mathrm{C}$ for Campinas, $21-30{ }^{\circ} \mathrm{C}$ for Bauru and $14-24{ }^{\circ} \mathrm{C}$ for Presidente Prudente). However, $59.5 \%$ of the total sample (308 out of 519 individuals) indicated comfort limits ranging from 18 to $26^{\circ} \mathrm{C}$, which is consistent with the limits proposed by Monteiro and Alucci for the city of Sao Paulo. These results can contribute to evaluate the thermal quality of other public spaces in the same towns.

Keywords: Open urban space. Thermal comfort. Pedestrian spaces. 


\section{Introdução}

As características ambientais de espaços públicos urbanos, que incluem aspectos físicos e microclimáticos, contribuem para torná-los mais ou menos atrativos à convivência humana. Esses espaços ganham significados de acordo com o contexto urbano e as características locais, que juntos irão determinar seus usos, seja de passagem ou de permanência. Assim, um espaço projetado para circulação de pessoas (espaço de passagem) pode ser transformado em espaço de convivência (permanência). Da mesma forma, espaços projetados para a permanência humana podem se tornar ambientes sem vida e sem utilidade (JACOBS, 196I; GEHL, 2010; SHAFTOE, 2008).

A dimensão da vida cotidiana pode ser avaliada mediante o uso de seus espaços públicos. A rua, por exemplo, projetada para mobilidade, passagem e interação entre pessoas, representa a espacialidade das relações sociais, através de suas formas e funções. Para algumas pessoas a rua é simplesmente passagem, de uso temporário, enquanto para outras ela pode significar mais que um itinerário. Entretanto, para que isso aconteça, o espaço público deve ser dotado de maior estrutura, equipamentos, segurança, além de qualidade ambiental, que inclui microclimas favoráveis ao conforto térmico e, consequentemente, ao convívio dos usuários.

Dessa forma, o entendimento das características do espaço urbano (geometria do espaço aberto, presença de água e de vegetação e de superfícies verdes em geral, e características das superfícies) que influenciam positiva ou negativamente os microclimas e o conforto térmico dos pedestres é imprescindível para subsidiar o planejamento e o projeto do espaço público. Nesse enfoque, observa-se um número crescente de pesquisas que objetivam a definição de requisitos ambientais úteis para o projeto urbano (Leveratto, 1999; Chrisomallidou et al., 2003; Nikolopoulou, 2004; Katzschner e Thorsson, 2009, Vasconcellos e Corbella, 2009, entre outros).

Entre esses estudos destacam-se aqui os relacionados à influência das variáveis ambientais no conforto térmico dos usuários de ruas, praças e parques. Nesses locais, o estado de conforto térmico é fortemente influenciado pela satisfação dos usuários, aspecto que torna muito complexa a definição dos limites de conforto nesses ambientes, pois envolve o entendimento da inter-relação entre numerosos e diferentes parâmetros.

De acordo com Nikolopoulou e Steemers (2003), uma aproximação quantitativa baseada apenas em parâmetros físicos, utilizados em modelos preditivos, não é suficiente para descrever as condições de conforto em espaços abertos. Por isso, esses autores chamam a atenção para a necessidade do entendimento da influência das oportunidades adaptativas (físicas, fisiológicas e psicológicas) na sensação de conforto térmico dos usuários. Entre elas, a adaptação psicológica é de extrema importância, pois os fatores comportamentais e psicológicos afetam a satisfação dos usuários e contribuem para diferenças existentes entre os votos de sensação real (ASV) e o conforto calculado por meio de índices preditivos.

Esses fatores contextuais, que incluem experiência térmica, percepção de controle térmico, cultura e duração de exposição, são coletivamente conhecidos como “adaptação térmica”, e a variação do conforto térmico com as estações do ano é um exemplo significativo desse tipo de adaptação (LIN; DE DEAR; HWANG, 2011). As experiências recentes dos usuários e suas expectativas desempenham um papel importante e são responsáveis por uma variação de até $10{ }^{\circ} \mathrm{C}$ na temperatura de conforto, que é a condição térmica em que as pessoas não sentem calor ou frio (NIKOLOPOULOU; LYKOUDIS, 2006).

Nesse contexto, observa-se uma variedade de estudos de caso, em diferentes países, objetivando investigar essas questões e verificar a aplicabilidade de diferentes índices preditivos de conforto térmico, contribuindo, assim, para sua calibração para a realidade local. No Brasil, as pesquisas desenvolvidas por Monteiro e Alucci (2006, 2007) inserem-se nesse contexto ao calibrar vários modelos preditivos de conforto e/ou estresse térmico, para a análise da adequação térmica de espaços abertos na cidade de São Paulo. Esses estudos têm servido de parâmetro de comparação com outros desenvolvidos no Estado de São Paulo, como as pesquisas de Shimakawa e BuenoBartholomei (2009), Dacanal et al. (2009), Brusantin e Fontes (2009), Fontes et al. (2010), que visaram analisar e comparar a sensação térmica real e a calculada por meio do índice preditivo de conforto temperatura fisiológica equivalente (PET).

O índice PET representa uma temperatura fictícia, resultante da interação entre variáveis físicas e ambientais de um ambiente real, e foi desenvolvido por Mayer e Höppe (1987) para ser aplicado tanto em ambientes externos como internos. A temperatura calculada, com base no modelo de balanço energético para indivíduos de Munique (MEMI), é equivalente a uma temperatura do ar de um ambiente interno típico,

168 Labaki, L. C.; Fontes, M. S. G. de C.; Bueno-Bartholomei, C. L.; Dacanal, C. 
onde a temperatura média radiante é igual à temperatura do ar; a velocidade do ar é definida como 0,1 m/s, e a pressão de vapor de água é de 12 hPa (HÖPPE, 1993, 1999). Atualmente, observa-se uma grande aplicação desse índice em pesquisas brasileiras, aspecto que pode ser atribuído, em parte, à facilidade de uso do software RayMan (MATZARAKIS; RUTZ; MAYER, 2006), desenvolvido pelo Instituto Meteorológico da Universidade de Freiburg (UNIVERSITÄT..., 2009).

Com o objetivo de caracterizar os espaços públicos de passagem e verificar como os aspectos físicos locais interferem no microclima e no conforto térmico de pedestres, esta pesquisa apresenta resultados de estudos de caso desenvolvidos em três cidades do Estado de São Paulo, Brasil. A metodologia inspirou-se no Projeto RUROS Rediscovering the Urban Realm and Open Space (NIKOLOPOULOU; LYKOUDIS, 2006). Para isso, foram selecionados três espaços de passagem nas cidades de Campinas, Bauru e Presidente Prudente, com características físicas e microclimas diversos.

\section{Caracterização das áreas de estudo}

As áreas de estudo estão localizadas nos centros das cidades de Campinas, Bauru e Presidente Prudente (Figura 1a e 1b), cujo percurso dos pedestres está associado com a presença de comércio, serviços e transportes públicos.
Portanto, essas áreas são caracterizadas por intenso fluxo de pedestres, muitas vezes em conflito com o tráfego de veículos.

De acordo com a classificação de Koeppen, o clima de Campinas (lat. 22,53으. long. $47,04^{\circ} \mathrm{W}$ ) é do tipo Cwa, caraterizado por verão chuvoso e inverno seco, com temperatura média do mês mais quente de $22{ }^{\circ} \mathrm{C}$. Já os climas de Bauru (lat. $22,36^{\circ} \mathrm{S}$, long. $49,03^{\circ} \mathrm{W}$ ) e Presidente Prudente (lat. $22,07^{\circ} \mathrm{S}$, long. $51,23^{\circ} \mathrm{W}$ ) são do tipo Aw, caracterizado por verão chuvoso e inverno seco, e temperatura média do mês mais frio de $18{ }^{\circ} \mathrm{C}$ (CENTRO..., 2011). A Figura 2 mostra as características climáticas mensais das três cidades, a partir de gráficos que apresentam valores máximos, mínimos e médios de temperatura do ar e dados de precipitação.

Em Campinas, a pesquisa foi realizada no Largo do Pará (Figura 3), um espaço projetado para permanência, mas que possui um uso intenso por pedestres, uma vez que serve de eixo de ligação de uma das principais avenidas da cidade, a av. Francisco Glicério, com as ruas comerciais do entorno, especialmente com uma rua de pedestres. O largo foi criado em meados do século XIX, ganhando o status de espaço público urbano no final do mesmo século, e suas funções mais importantes estão associadas com a presença de transportes públicos (regional e municipal), táxi, hotéis e comércio no bairro.

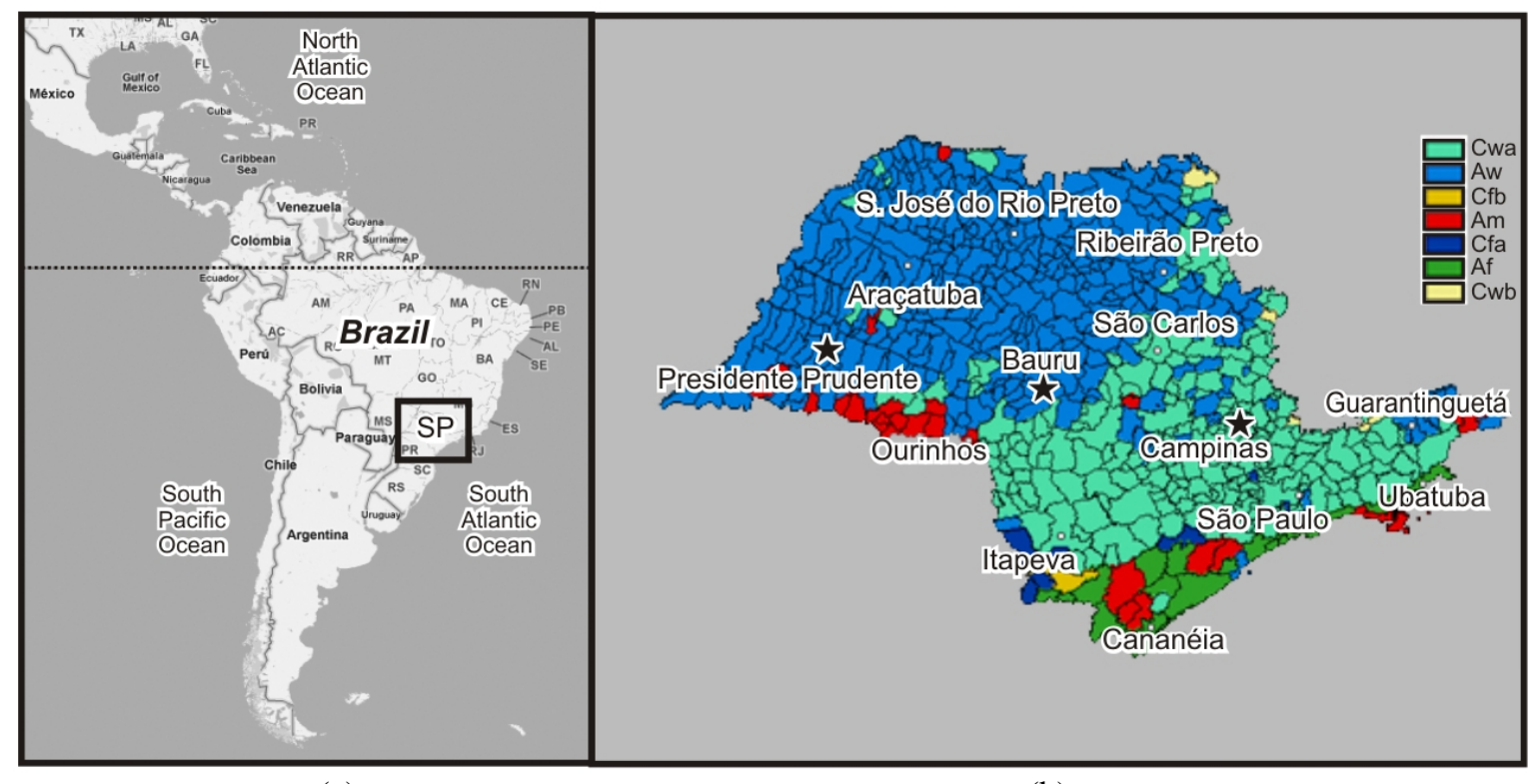

(a)

(b)

Figura 1 - (a) Mapa da América do Sul com destaque para o Estado de São Paulo; (b) Mapa do Estado de São Paulo com a classificação climática de Koeppen, indicando as cidades de estudo (marcadas com uma estrela)

Fonte: Centro de Pesquisas Meteorológicas e Climáticas aplicadas à Agricultura - CEPAGRI (2011). 


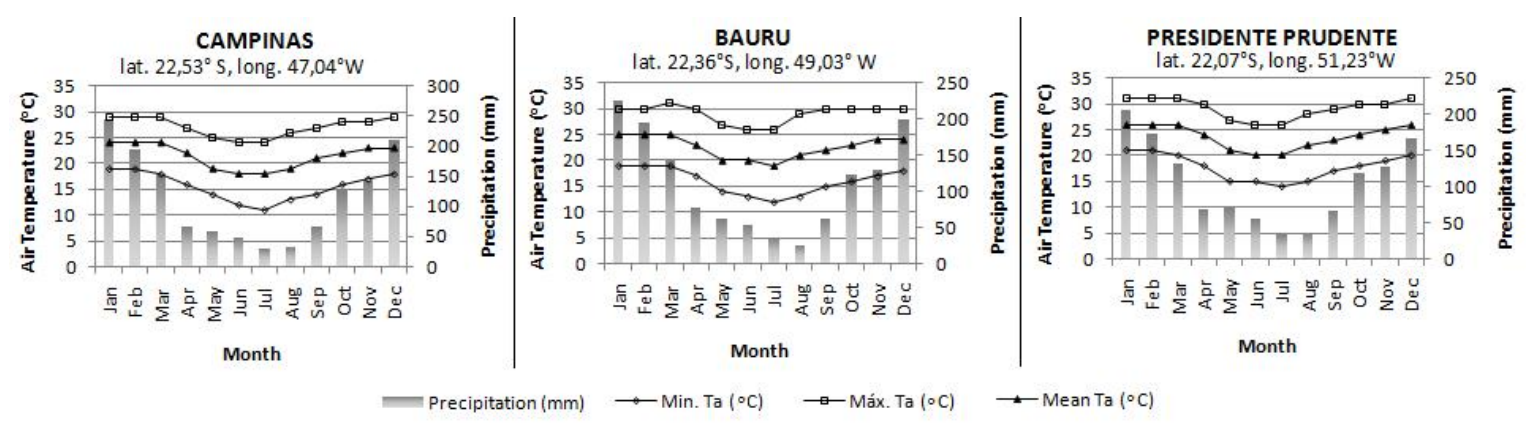

Figura 2 - Valores médios mensais de temperaturas máxima, mínima e média e de precipitação nas cidades de Campinas, Bauru e Presidente Prudente

Fonte: Centro de Pesquisas Meteorológicas e Climáticas aplicadas à Agricultura - CEPAGRI (2011).

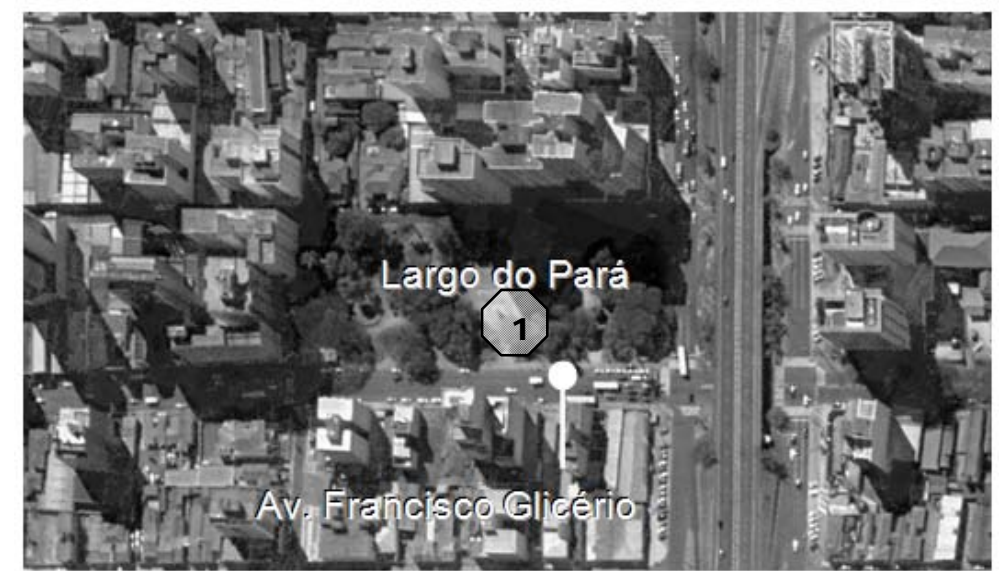

Figura 3 - Largo do Pará, em Campinas, SP

Fonte: adaptado do Google Earth (2009).

O largo possui equipamentos como playground, comércio de alimentos e banca de jornal; é cercado por edifícios de dois até dez andares, pintados de diversas cores; as calçadas são constituídas de pedra portuguesa; os jardins são bem definidos e possuem várias espécies de plantas de diferentes tamanhos; os bancos são feitos de madeira e ferro fundido; outros mobiliários são de concreto e de ferro fundido; os equipamentos do playground são de madeira com piso de areia, localizados em área limitada por cerca de ferro.

Em Bauru, o estudo de caso foi realizado na rua de pedestres Batista de Carvalho (Figura 4), de importância histórica, uma vez que foi o portão de entrada na cidade para os passageiros que desembarcavam na estação ferroviária viajando pela empresa Noroeste do Brasil. Após o declínio do transporte ferroviário no Brasil, a rua tornou-se um ponto de atração para o comércio e possui impacto regional. Em 1992, seu uso ficou restrito aos pedestres, época em que foram implantados equipamentos públicos como bancos, lixeiras, luminárias, pórticos, entre outros.
Os edifícios comerciais que servem de fronteira são constituídos de alvenaria de tijolo, possuem, em sua maioria, dois a três pavimentos e são pintados em cores diferentes. A base é constituída de pedra portuguesa nas cores branca e preta; o mobiliário urbano é feito de madeira e concreto (bancos e canteiros) e possui pórticos de estrutura metálica; alguns deles possuem cobertura de policarbonato transparente na cor azul.

Em Presidente Prudente o estudo foi realizado na rua Nicolau Maffei (Figura 5), de uso exclusivo de pedestres desde o início dos anos 80. O intenso fluxo de pedestres local deve-se especialmente ao uso do comércio, serviços de bancos ou mesmo como espaço de passagem para realizar outras atividades. Os equipamentos urbanos implantados nessa rua são bancos, cabines de telefone público, lixeiras, canteiros de flores e mesas e cadeiras das lojas de alimentos. A rua, sombreada por árvores e edifícios de alvenaria de tijolo e pintados com cores variadas, possui piso de pedra portuguesa e bancos e canteiros de concreto.

170 Labaki, L. C.; Fontes, M. S. G. de C.; Bueno-Bartholomei, C. L.; Dacanal, C. 


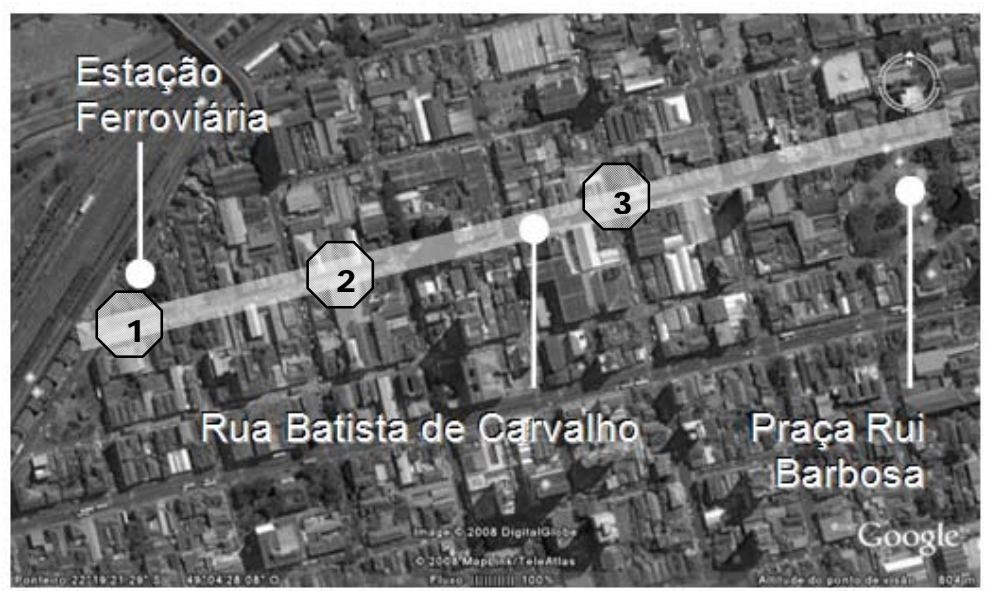

Figura 4 - Rua Batista de Carvalho, em Bauru, SP

Fonte: adaptado do Google Earth (2009).

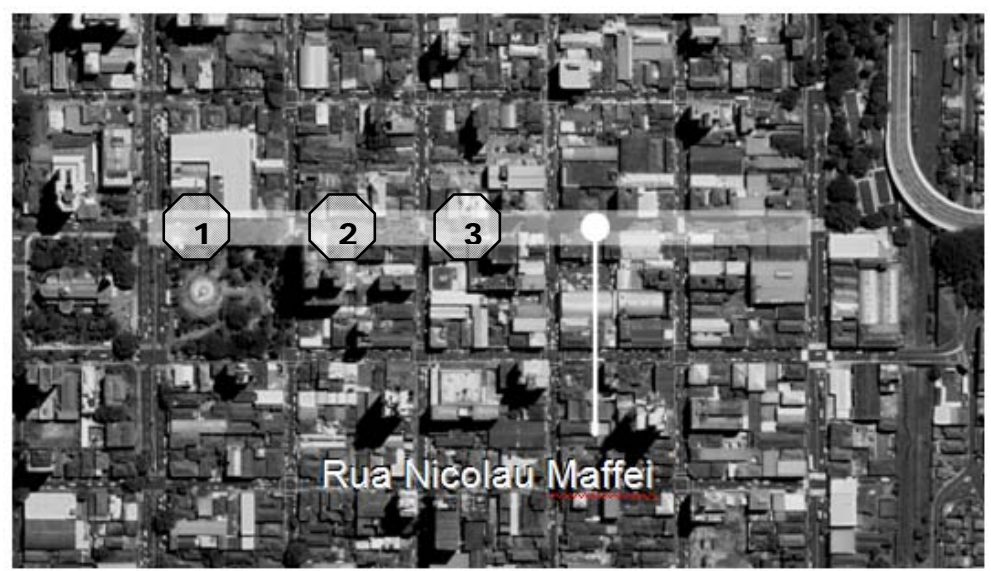

Figura 5 - Rua Nicolau Maffei, em Presidente Prudente

Fonte: adaptado do Google Earth (2009).

Os três espaços analisados possuem algumas características em comum, sendo uma delas a utilização da pedra portuguesa como material de revestimento da base. Essa pedra possui composição de calcário e basalto, com alta condutividade térmica (1,6 a 2,9 W/mK) e é amplamente utilizada para pavimentação de praças e ruas de pedestres, em razão da flexibilidade de composição de desenhos e manutenção. Outras características encontradas foram:

(a) a utilização do concreto e madeira no mobiliário urbano;

(b) os materiais dos edifícios fronteiriços, constituídos de alvenaria de tijolos (condutividade térmica de 1,6 e 2,9 W/mK); e

(c) as cores das fachadas (absortância entre 0,20 e 0,97 e emissividade 0,9 ).
A Figura 6 apresenta os pontos de monitoramento dos dados, juntamente com as cartas solares de cada cidade sobrepostas a fotografias hemisféricas, as quais permitem uma visualização da influência da geometria urbana e da arborização no fator de visão do céu (SVF) e, consequentemente, nos períodos de incidência da radiação solar direta de cada local.

\section{Materiais e métodos}

A investigação sobre as características microclimáticas e condições de conforto térmico nos três espaços públicos descritos foi realizada em diferentes condições de tempo (frio e seco, quente e seco, e quente e úmido), durante seis dias em cada uma das cidades, no período de setembro de 2008 a março de 2009. 


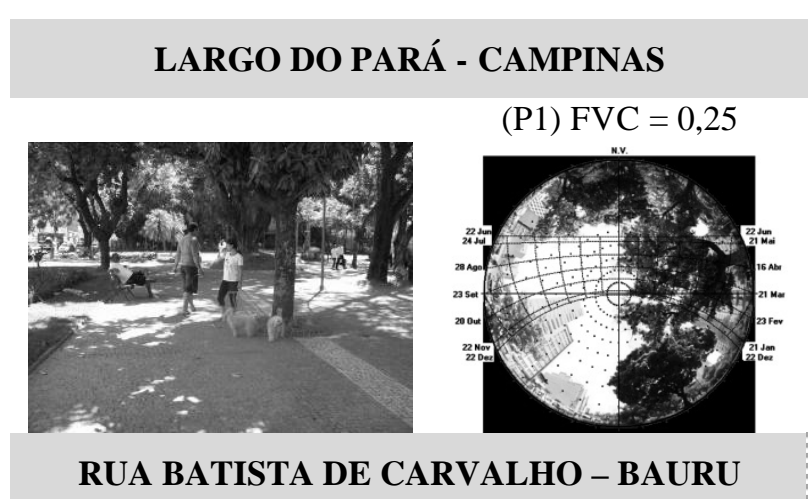

(P1) $\mathrm{FVC}=0,40$
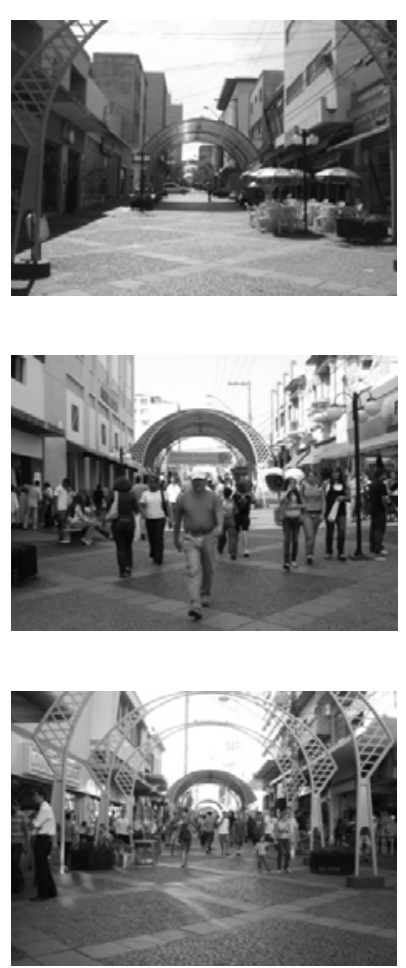

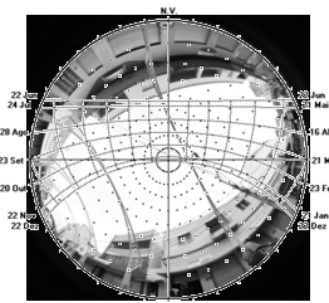

(P2) $\mathrm{FVC}=0,26$

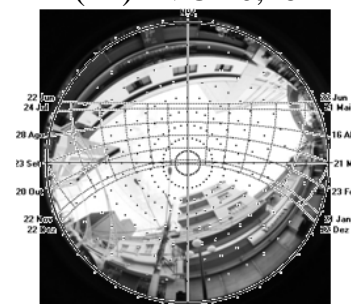

(P3) FVC=0,31

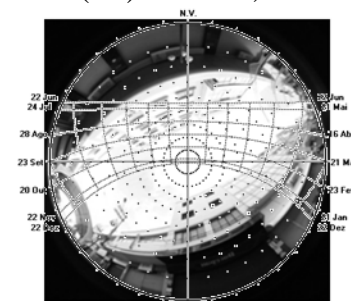

\section{RUA NICOLAU MAFFEY - PRESIDENTE} PRUDENTE

(P1) $\mathrm{FVC}=0,15$
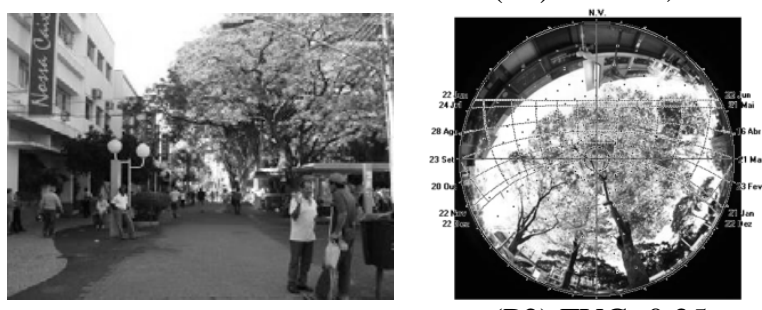

(P2) $\mathrm{FVC}=0,25$
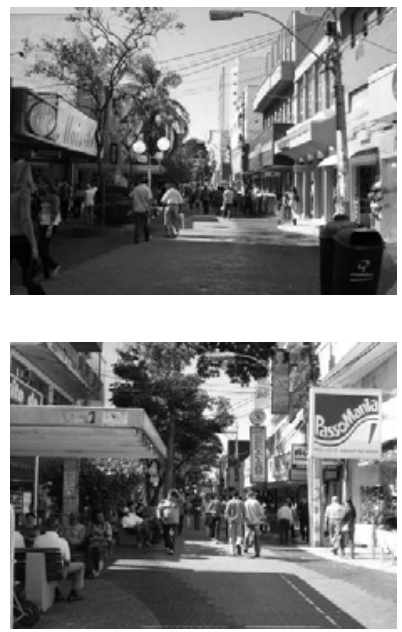

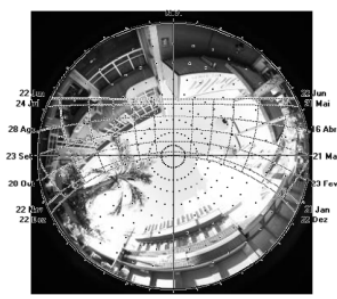

(P3) FVC=0,15

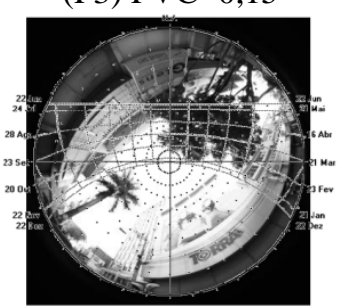

Figura 6 - Pontos de monitoramento microclimático e fatores de visão do céu (FVC) correspondentes

Os levantamentos de dados microclimáticos (temperatura do ar e de globo, umidade relativa do ar, velocidade do vento e radiação solar global) foram coletados em uma estação meteorológica móvel (Figura 7), especialmente projetada para a pesquisa por técnicos do Laboratório de Conforto Ambiental e Física Aplicada da Universidade Estadual de Campinas (Unicamp). Os instrumentos foram fixados a 1,5 m acima do solo, e o Net radiômetro foi direcionado para o norte, conforme instrução do fabricante, com o pirgeômetro e o piranômetro deslocados a $0,5 \mathrm{~m}$ da estação. Para estabilização dos instrumentos esperaram-se 15 min, para então dar-se início à coleta de dados em intervalos de $1 \mathrm{~min}$.
Simultaneamente à aquisição de dados microclimáticos, foram aplicados questionários em uma amostra definida em função do uso semanal dos espaços em cada cidade (total de 519 indivíduos). Os usuários foram escolhidos aleatoriamente, objetivando identificar seu perfil (idade, peso, altura, atividade desenvolvida, isolamento térmico da roupa), votos de sensação real (ASV), preferência e satisfação térmica, motivos e frequência de uso do espaço, além de questões que afetam os usos. O questionário aplicado teve como base o desenvolvido no Projeto RUROS (NIKOLOPOULOU; LYKOUDIS, 2006). A Figura 8 mostra parte das questões relacionadas à identificação da sensação, percepção (preferência térmica) e satisfação dos usuários (resposta "sim" ou “não" para a questão "Você se sente

172 Labaki, L. C.; Fontes, M. S. G. de C.; Bueno-Bartholomei, C. L.; Dacanal, C. 
confortável?”). Em relação à questão sobre a sensação térmica ("o que você acha do microclima local”) foram necessárias explicações para que os usuários declarassem sua sensação em relação às condições térmicas locais.

Os dados coletados em campo permitiram identificar o índice de conforto PET e os ASV, através da escala de sensação térmica de cinco pontos para o ASV, que varia de -2 a 2 (muito frio, frio, nem frio nem quente, quente e muito quente), adotada por Nikolopoulos e Steemers (2003). O índice PET foi calculado pelo software RayMan (versão 1.2), desenvolvido pelo Meteorological Institute of Freiburg (MATZARAKIS et al., 2007), que permite a inserção dos seguintes dados dos usuários entrevistados: atividade metabólica (W), resistência térmica das roupas (clo), sexo (m, f), idade, peso $(\mathrm{kg})$ e altura $(\mathrm{m})$, além de dados microclimáticos de temperatura do ar $\left({ }^{\circ} \mathrm{C}\right)$, umidade relativa do ar (\%) ou pressão de vapor, velocidade do ar $(\mathrm{m} / \mathrm{s})$ e radiação global $\left(\mathrm{W} / \mathrm{m}^{2}\right)$ ou temperatura radiante média $\left({ }^{\circ} \mathrm{C}\right)$.

Em cada ponto de medição foi tirada uma fotografia hemisférica utilizando-se uma câmera Canon XSI Rebel equipada com lentes olho de peixe Canon 180 EX Sigma. As imagens foram processadas para melhorar o contraste e brilho, e convertidas em escala de cinza, para destacar o céu e os outros elementos do espaço físico. O SVF foi obtido por meio do software RayMan 1.2.

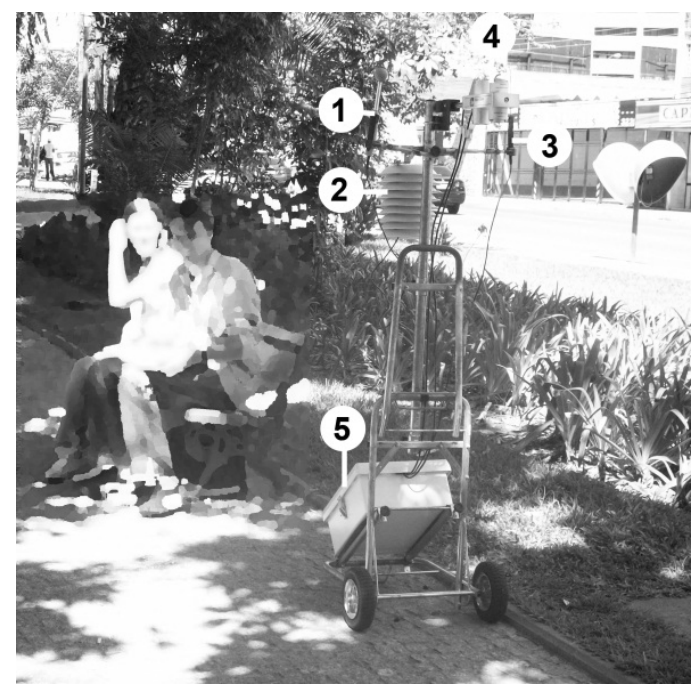

Figura 7 - Estação meteorológica móvel

Nota: Legenda:

1. Sensor de temperatura de globo (Modelo I: 0613 1712; fabricante: Testo), construído com bola de pinguepongue oficial pintada na cor cinza-burguês (emissividade 0,9 , refletância solar 0,3 ).

2. Datalogger de temperatura e umidade (Modelo: 175-H1 da Testo).

3. Anemômetro omnidirecional, de esfera aquecida ( $\varnothing 3 \mathrm{~mm}$; Modelo: 0635 1549; fabricante: Testo).

4. Net radiômetro (Fabricante: Kipp \& Zonen)

5. Datalogger (Modelo: CR1000; Fabricante: Campbell Scientific).

No momento, o que você acha do microclima local (sensação térmica):

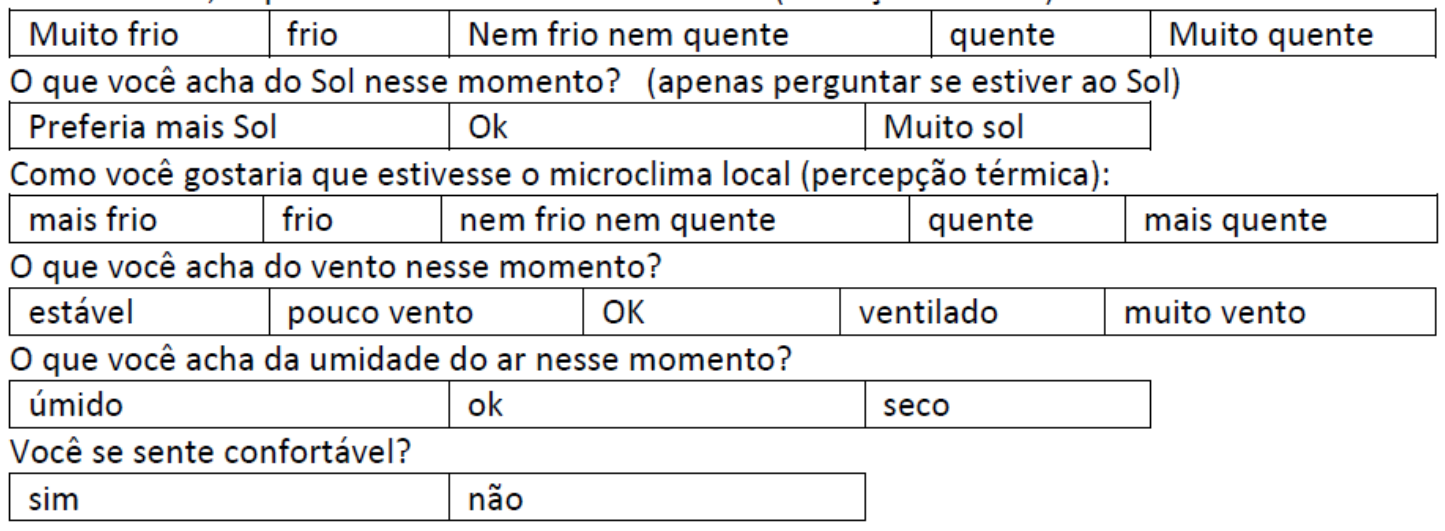

Figura 8 - Questões para identificação da sensação, preferência e satisfação térmica dos usuários 


\section{Resultados}

A análise das condições de conforto térmico em espaços públicos de passagem nas cidades de Campinas, Bauru e Presidente Prudente permitiu evidenciar grande sensibilidade térmica dos usuários às variações diárias e sazonais das condições do tempo. A Tabela 1 apresenta dados microclimáticos médios durante a pesquisa de campo nas três cidades, os quais mostram diferenças microclimáticas nos períodos da manhã e da tarde. Em condições de tempo ameno (frio e seco), observou-se uma quantidade significativa de usuários confortáveis (Figura 9) e maior equilíbrio entre o conforto real e o calculado. Para essas condições, os limites de conforto calculado (através do índice PET) estiveram entre 18 e $23^{\circ} \mathrm{C}$.

Tabela 1 - Condições microclimáticas durante a pesquisa de campo nas cidades de Campinas, Bauru e Presidente Prudente

\begin{tabular}{|c|c|c|c|c|c|c|}
\hline \multirow[t]{2}{*}{ Cidades } & \multicolumn{2}{|c|}{ Campinas } & \multicolumn{2}{|c|}{ Bauru } & \multicolumn{2}{|c|}{ Presidente Prudente } \\
\hline & $\mathbf{M}$ & $\mathbf{T}$ & $\mathbf{M}$ & $\mathbf{T}$ & $\mathbf{M}$ & $\mathbf{T}$ \\
\hline \multicolumn{7}{|c|}{ Temperatura $\left({ }^{\circ} \mathrm{C}\right)$} \\
\hline Média & 25,1 & 27,7 & 26,8 & 29,9 & 16,0 & 28,0 \\
\hline Mmín & 21,7 & 26,1 & 22,7 & 25,4 & 10,0 & 22,5 \\
\hline Mmáx & 28,2 & 30,1 & 30,5 & 35,2 & 24,6 & 32,6 \\
\hline \multicolumn{7}{|c|}{ Umidade relativa do ar (\%) } \\
\hline Média & 52,5 & 51,5 & 52,5 & 40,5 & 64,6 & 41,3 \\
\hline Mmín & 44,7 & 41,6 & 41,9 & 28,2 & 52,2 & 37,1 \\
\hline Mmáx & 58,3 & 62,8 & 62,4 & 59,9 & 79,1 & 48,9 \\
\hline \multicolumn{7}{|c|}{ Velocidade dos ventos (m/s) } \\
\hline Média & 0,7 & 0,8 & 1,1 & 1,2 & 1,1 & 1,0 \\
\hline Mmín & 0,2 & 0,2 & 0,7 & 0,8 & 0,7 & 0,9 \\
\hline Mmáx & 1,0 & 1,3 & 1,9 & 2,1 & 1,7 & 1,2 \\
\hline & \multicolumn{6}{|c|}{ Radiação solar global $\left(\mathrm{W} / \mathrm{m}^{2}\right)$} \\
\hline Mmín & -95 & -383 & 304 & 40 & 50 & -33 \\
\hline Mmáx & 270 & 462 & 562 & 282 & 408 & 549 \\
\hline
\end{tabular}

Nota: Legenda:
M - Manhã:
T - Tarde;
Mmín - média mínima; e
Mmáx - Média máxima.

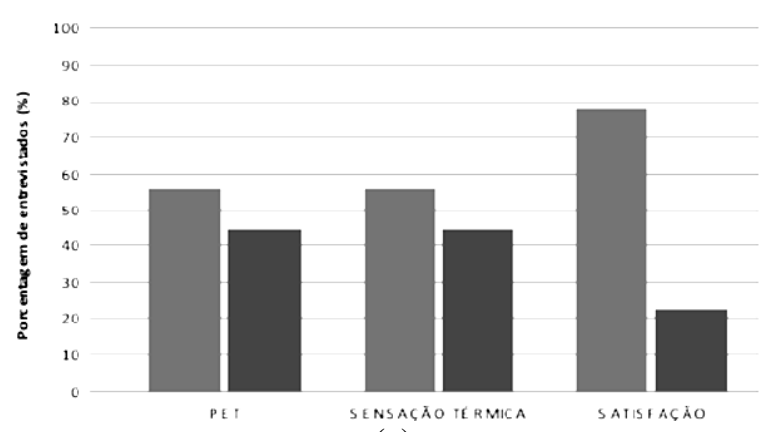

(a)

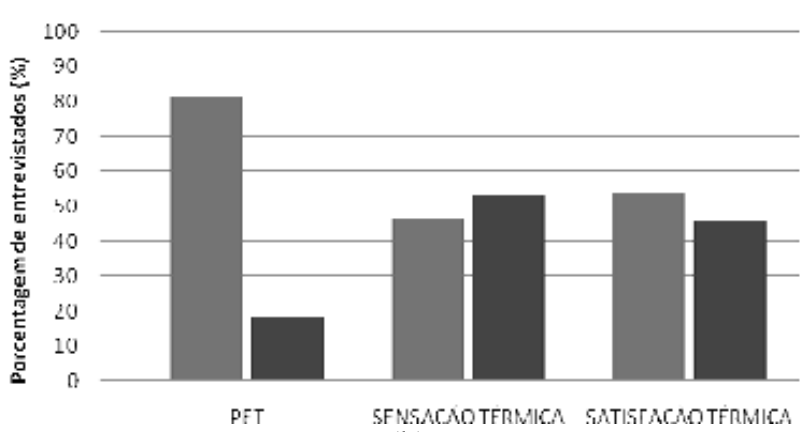

(b)

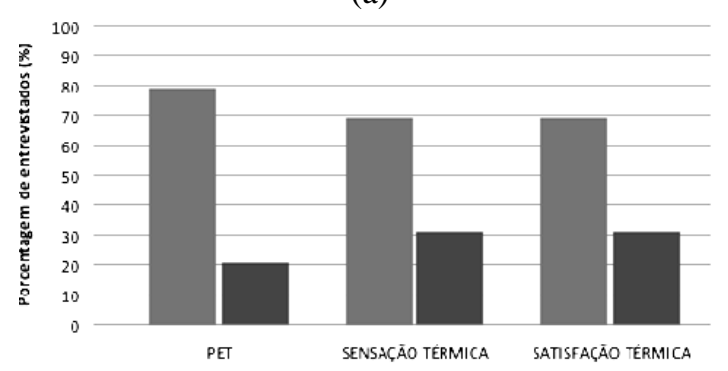

(c)

Figura 9 - Comparação entre o conforto real e o calculado em condições de tempo frio e seco nas cidades de (a) Campinas, (b) Bauru e (c) Presidente Prudente

Nota: Legenda: $\quad$ Confortável $\quad$ Desconfortável

174 Labaki, L. C.; Fontes, M. S. G. de C.; Bueno-Bartholomei, C. L.; Dacanal, C. 
Já em condições de tempo quente e úmido (manhã) e quente e seco (tarde), verificou-se maior quantidade de usuários desconfortáveis, tanto em relação ao conforto real quanto ao calculado. Em alguns períodos em que as temperaturas atingiram valores superiores a $30{ }^{\circ} \mathrm{C}$, a porcentagem de usuários desconfortáveis em relação à temperatura PET atingiu 100\%, especialmente nas cidades de Bauru e Presidente Prudente (Figura 10). Nessas condições de grande desconforto, evidenciadas especialmente nos períodos da tarde, em virtude das temperaturas elevadas associadas à alta ou baixa umidade relativa do ar, muitos usuários recusaram-se a responder aos questionários alegando a necessidade de sair do local o mais rapidamente possível. Outros, porém, ao serem entrevistados, apontaram possíveis soluções para mitigar as condições adversas locais.

A grande sensibilidade térmica dos usuários em espaços de passagem contrasta com estudo similar desenvolvido pelos autores (FONTES et al., 2010) em espaços de convivência (permanência). Nesses espaços, o desempenho ambiental, associado aos aspectos de circulação, atividades e presença de nichos, interferiu significativamente na percepção de conforto térmico dos usuários, que de uma maneira geral declararam-se confortáveis, mesmo em condições de tempo adversas.

A Figura 11 apresenta gráficos do tipo boxplot para todos os valores de temperatura PET agrupados de acordo com os ASV, nas cidades de Campinas, Bauru e Presidente Prudente

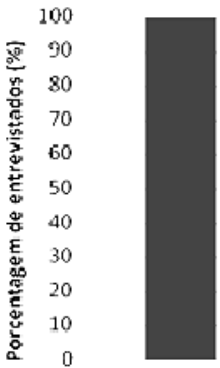

PET

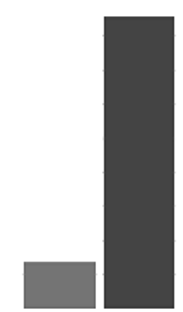

SENSACAOTERMICA SATISFACAOTERMICA respectivamente. Nos gráficos, as barras verticais indicam a porcentagem de $50 \%$ dos entrevistados para cada voto de sensação, a partir de um valor mediano. Assim, os valores de neutralidade para a temperatura PET variaram de 20 a $29{ }^{\circ} \mathrm{C}$ em Campinas, de 21 a $30{ }^{\circ} \mathrm{C}$ em Bauru, e de 14 a 24 ${ }^{\circ} \mathrm{C}$ em Presidente Prudente.

A Figura 12 apresenta os gráficos considerando-se os resultados para todos os espaços de passagem e relaciona os intervalos de temperatura PET aos ASV. Verifica-se que, de um total de 519 entrevistados, 235 declararam que a sensação térmica era "nem frio nem quente". A temperatura PET para essas pessoas mostrou grande variação, mas o intervalo que inclui $50 \%$ dos indivíduos variou de 17,5 a $25,3{ }^{\circ} \mathrm{C}$. Porém, assim como nas análises individuais para cada cidade, essa faixa sobrepõe-se a outros intervalos que indicariam desconforto, seja por frio ou calor.

Se forem considerados os limites de temperatura PET para a neutralidade térmica entre 18 e $26^{\circ} \mathrm{C}$, de acordo com Monteiro e Alucci (2007), encontra-se 59,5\% da amostra total (308 de 519 indivíduos). Entretanto, parte considerável desses 308 respondentes declarou como sensação térmica “quente" (18,5\%) ou "frio" (17,5\%). Análise semelhante sobre espaços verdes públicos nas mesmas cidades do Estado de São Paulo, apresentada em Fontes et al. (2010), permitiu identificar $70 \%$ de todos os indivíduos em neutralidade térmica dentro desses limites.

Figura 10 - Comparação entre o conforto real e o calculado em condições de tempo quente e úmido nas cidades de Bauru e Presidente Prudente

Nota: Legenda: $\quad$ Confortável $\quad$ Desconfortável 


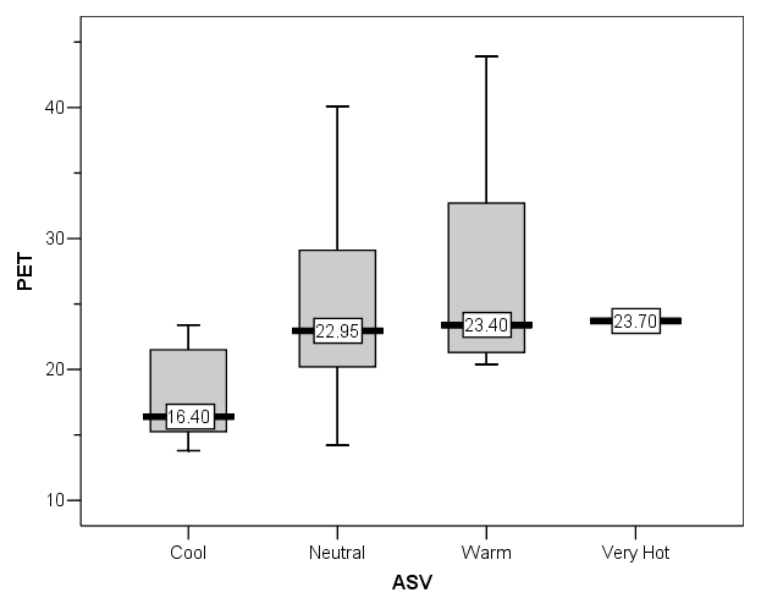

(a)

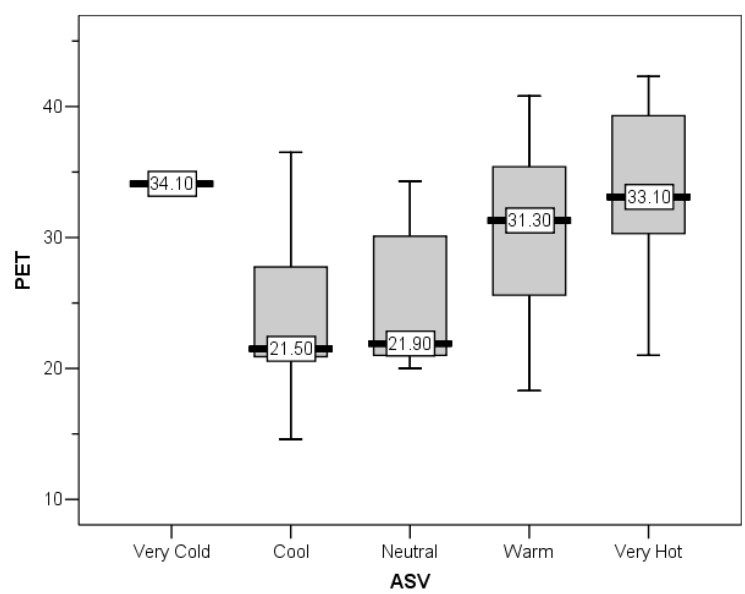

(b)

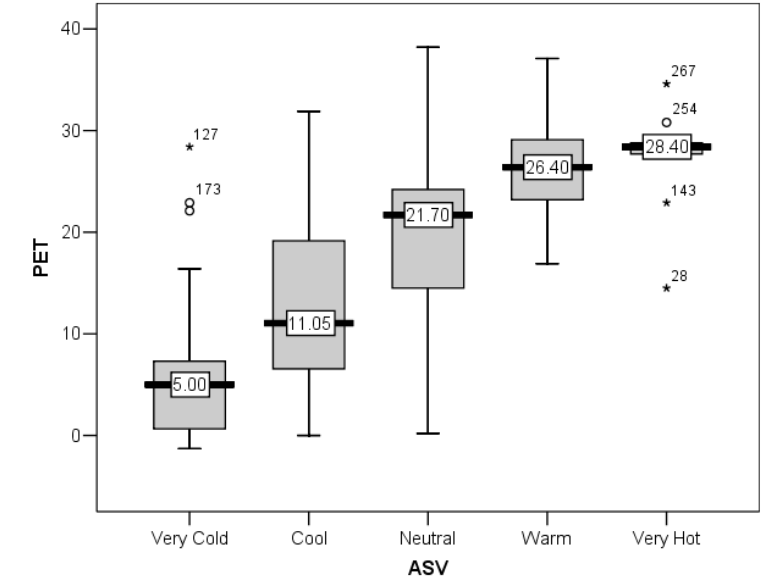

(c)

Figura 11 - Gráficos (PET x ASV) com a faixa de conforto térmico para espaço de passagem em (a) Campinas, (b) Bauru, e (c) Presidente Prudente

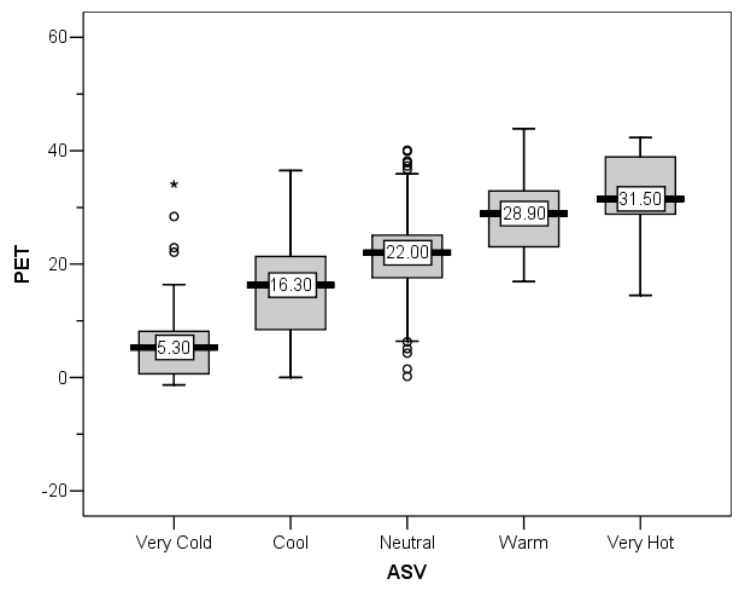

Figura 12 - Faixa de temperatura PET relacionada às faixas de sensação térmica nas três cidades analisadas 


\section{Conclusão}

A análise das condições de conforto térmico em espaços públicos de passagem em três cidades do Estado de São Paulo, além de contribuir para o avanço das pesquisas na área no país, evidenciou grande sensibilidade térmica em relação às variações diárias e sazonais, assim como diferentes limites para o PET de neutralidade térmica para cada um dos espaços estudados. As maiores diferenças entre o conforto térmico real e o calculado foram observadas em condições de tempo ameno (frio e seco), ao passo que as semelhanças apareceram em condições de tempo quente (seco ou úmido).

No primeiro caso, o conforto calculado identificou uma maior quantidade de pessoas confortáveis, enquanto o ASV mostrou um equilíbrio entre pessoas confortáveis e desconfortáveis. No segundo, observou-se uma maior quantidade de pessoas desconfortáveis, em qualquer uma das duas formas de avaliação do conforto térmico e em cada uma das cidades. Esses resultados mostram que a tolerância para as condições de tempo quente é menor para os usuários de espaços de passagem do que para os espaços de permanência arborizados (FONTES et al., 2010). Esse aspecto evidencia a necessidade de uma atenção especial para subsidiar novos projetos ou requalificar espaços semelhantes, objetivando melhorar suas condições microclimáticas e, consequentemente, otimizar o conforto térmico de seus usuários.

Apesar dos diferentes limites de temperatura PET para a neutralidade térmica em cada cidade, 59,5\% de todos os indivíduos confortáveis (308 de 519) estiveram inseridos na faixa de $18-26{ }^{\circ} \mathrm{C}$, ou seja, em conformidade com os limites de conforto para o PET propostos por Monteiro e Alucci (2007) para a cidade de São Paulo, ajustados em relação à faixa de $18-23{ }^{\circ} \mathrm{C}$ proposta por Matzarakis, Rutz e Mayer (2000). Por isso, a faixa de $18-26{ }^{\circ} \mathrm{C}$ pode ser adotada como parâmetro de avaliação da qualidade térmica de espaços de passagem nas cidades analisadas, mas novos estudos são necessários para a corroboração desses valores.

Para estudos similares, recomenda-se a expansão da escala de votos de sensação real (ASV), evitando-se, assim, a sobreposição dos votos em cada caso analisado, adotando-se intervalos de valores contínuos $(-3 \mathrm{a}+3$, zero como neutralidade térmica), para substituir a escala de cinco pontos empregada neste trabalho.

\section{Referências}

BRUSANTIN, G. N.; FONTES, M. S. G. C. Conforto Térmico em Espaços Públicos de Permanência: uma experiência na cidade de BauruSP. In: ENCONTRO NACIONAL, 10.; ENCONTRO LATINO-AMERICANO DE CONFORTO NO AMBIENTE CONSTRUÍDO, 6., Natal, 2009. Anais... Porto Alegre: ANTAC, 2009. p. 441-449.

\section{CENTRO DE PESQUISAS}

METEOROLÓGICAS E CLIMÁTICAS

APLICADAS A AGRICULTURA. [Clima dos

Municípios Paulistas]. Disponível em:

$<$ http://www.cpa.unicamp.br>. Acesso em: 4 mar. 2011.

CHRISOMALLIDOU, N. et al. Rehabilitation of Open Space Under Bioclimatic Criteria. In: CONFERENCE ON PASSIVE AND LOW ENERGY ARCHITECTURE, 20., Santiago, 2003. Proceedings... Santiago: Universidad Católica do Chile, 2003.

DACANAL, C. et al. Conforto Térmico em Espaços Livres Públicos: estudo de caso em Campinas, SP. In: ENCONTRO NACIONAL, 10.; ENCONTRO LATINO AMERICANO DE CONFORTO NO AMBIENTE CONSTRUÍDO, 6., Natal, 2009. Anais... Porto Alegre: ANTAC, 2009. p. 563-572.

FONTES, M. S. G. F. et al. Thermal Comfort in Open Public Spaces: studies in green areas in cities of the Sao Paulo State, Brazil. In:

INTERNATIONAL CONFERENCE ON

PASSIVE AND LOW ENERGY COOLING FOR THE BUILT ENVIRONMENT, 3., Rhodes, 2010. Proceedings... Rhodes, 2010.

GEHL, J. Cities for People. Washington: Island Press, 2010. 269 p.

GOOGLE EARTH. [Imagens]. 2009. Disponível em: <http://www.google.com/intl/ptPT/earth/index.html>. Acesso em: 11 out. 2011.

HÖPPE, P. Heat Balance Modelling. Experientia, Basel, v. 49, n. 9, p. 741-746, 1993.

HÖPPE, P. The Physiological Equivalent Temperature: a universal index for the biometeorological assessment of the thermal environment. International Journal of Biometeorology, Lisse, v. 43, n. 2, p. 71-75, 1999.

JACOBS, J. The Death and Life of Great

American Cities. London: Penguin Books, 1961, $221 \mathrm{p}$. 
KATZSCHNER, L.; THORSSON, S. Microclimatic Investigations as Tool for Urban Design. In: INTERNATIONAL CONFERENCE ON URBAN CLIMATE, 7., Yokohama, Japan, 2009. Proceedings... Yokohama, Japan, 2009.

LEVERATTO, M. J. Propuesta de Un Método para Analisar las Condiciones Microclimáticas en Espacios Urbanos. In: ENCONTRO NACIONAL, 5., ENCONTRO LATINO AMERICANO DE CONFORTO NO AMBIENTE CONSTRUÍDO, 2., Fortaleza, 1999. Anais... Porto Alegre: ANTAC, 1999.

LIN, T. P.; DE DEAR, R.; HWANG, R. L. Effect of Thermal Adaptation on Seasonal Outdoor Thermal Comfort. International Journal of Climatology, v. 31, n. 2, p. 302-312, 2011.

MAYER, H.; HÖPPE, P. Thermal Comfort of Man in Different Uurban environments. Theoretical and Applied Climatology, v. 38, p. 43-49, 1987.

MATZARAKIS, A.; RUTZ, F.; MAYER, H. Estimation and Calculation of the Mean Radiant Temperature Within Urban Structures. In: DE DEAR, R. J. et al. (Eds.). Biometeorology and Urban Climatology at the Turn of the Millenium: selected papers the conference ICBICUC'99. Sydney: WCASP-50, WMO/TD, 2000.

MATZARAKIS, A.; RUTZ, F.; MAYER, H. Modelling the thermal bioclimate in urban areas with the RayMan Model. In: INTERNATIONAL CONFERENCE ON PASSIVE AND LOW ENERGY ARCHITECTURE, 23., 2006, Genève. Proceedings... Genève: Université de Genève, 2006. v. 2, p. 449-453.

MATZARAKIS A, RUTZ F, MAYER H. Modelling radiation Fluxes in Simple and Complex Environments: application of the RayMan model. International Journal of Biometeorology, v. 51, p. 323-334, 2007.

MONTEIRO, L. M; ALUCCI, M. P. Calibration of outdoor thermal comfort models. In: INTERNATIONAL CONFERENCE ON PASSIVE AND LOW ENERGY ARCHITECTURE, 23, 2006. Proceedings... Genève: Université de Genève, 2006: 515-522.

MONTEIRO, L. M.; ALUCCI, M. P. Conforto Térmico em Espaços Abertos com Diferentes Abrangências Microclimáticas: parte 2: proposição de calibração de modelos preditivos. In: ENCONTRO NACIONAL, 9., ENCONTRO LATINO AMERICANO DE CONFORTO NO AMBIENTE CONSTRUÍDO, 5., 2007, Ouro Preto. Anais... Porto Alegre: ANTAC, 2007. p. 1231-1240.
NIKOLOPOULOU, M.; STEEMERS, K. Thermal Comfort and Psychological Adaptation as a Guide For Designing Urban Spaces. Energy and Buildings, v. 35, n. 1, p. 95-101, jan. 2003. NIKOLOPOULOU, M. (Org.). Designing Open Spaces in Urban Environment: a bioclimatic approach. Greece: Centre for renewable Energy Sources (C.R.E.S.), 2004.

NIKOLOPOULOU, M.; LYKOUDIS, S. Thermal Comfort in Outdoor Urban Spaces: analysis across different European countries. Building and Environment, Oxford, v. 41, n. 11, p. 1455-1470, nov. 2006.

SHAFTOE, H. Convivial Urban spaces: creating effective public places. London: Earthscan, 2008. $154 \mathrm{p}$.

SHIMAKAWA, A. H.; BUENO-

BARTHOLOMEI, C. L. Aplicação dos Modelos Preditivos de Conforto PET e PMV em Presidente Prudente - SP: estudo de caso: Parque do Povo. In: ENCONTRO NACIONAL, 10., ENCONTRO LATINO AMERICANO DE CONFORTO NO AMBIENTE CONSTRUÍDO, 6., 2009, Natal. Anais... Porto Alegre: ANTAC, 2009. p. 543-552.

VASCONCELLOS, V. M. N.; CORBELLA, O. D. Entorno construído e microclima: uma contribuição metodológica para o estudo de praças em cidade tropical quente e úmida. In: ENCONTRO NACIONAL, 10, ENCONTRO LATINO AMERICANO SOBRE CONFORTO NO AMBIENTE CONSTRUÍDO, 6., 2009, Natal. Anais... Porto Alegre: ANTAC, 2009, p.19631972.

UNIVERSITÄT FREIBURG. Meteorologisches Institut. RayMan Version 1.2. Freiburg, 2009. Disponível em:

http://www.urbanclimate.net/rayman/index.htm. Acesso em: 1 mar. 2009.

\section{Agradecimentos}

Os autores agradecem à Fundação de Amparo à Pesquisa do Estado de São Paulo (Fapesp), pelo auxílio-pesquisa recebido (Processo 2007/005634), e à Capes, pela bolsa de doutorado (C.D).

178 Labaki, L. C.; Fontes, M. S. G. de C.; Bueno-Bartholomei, C. L.; Dacanal, C. 